\title{
VARIAÇÃO DE CERTAS CARACTERISTICAS DE RAMI EM FUNÇÃO DA NATUREZA DA MEDULA DO CAULE (1)
}

Guilherme Augusto de Paiva Castro (2), Antonio luiz de Barros Salgado (2), Seção de Plantas Fibrosas, e Violeta NAGAI (2), Seçāo de Técnica Experimental e Cálculo, Instituto Agronómico

\section{SINOPSE}

Estudaram-se algumas características de dez variedades de rami (Boehmeria nivea Gaud.) em relação à presença ou à ausência do tecido medular do caule.

As variedades apresentaram porcentagens diferentes de número de caules com presença (cheios) ou ausência (ocos) de tecido medular.

Algumas mostraram superioridade dos caules cheios em relação aos caules ocos para as caracteristicas de peso, porcentagem de fibra sobre peso de caule verde, diâmetro e comprimento de caule, porém näo apresentaram diferenças para as características de porcentagem de fibra sobre peso de caule seco e comprimento de fibra.

Para fineza de fibra, somente a variedade tatsuka-kairyo revelou uma superioridade dos caules ocos em relação aos cheios.

Considerando-se os caules cheios e os ocos separadamente para cada variedade, as variedades, comparadas duas a duas, tiveram comportamento diferente com relação às características estudadas.

As variedades saikeiseishin-shun, E 49-63 o tatsuka-kairyo, tanto para caules cheios como para ocos, revelaram os maiores valores para as características estudadas, rom exceção para fineza de fibra, cuios valores foram os mais baixos, devendo ser escolhidas em futuros trabalhos de melhoramento.

Pelos resultados obtidos, verificou-se que é possível a realização de um trabalho de melhoramento visando aperfeiçoar determinadas características de rami

\section{INTRODUÇÃO}

Em um estudo de dez variedades de rami com a finalidade de determinar algumas das suas características principais (1), notaram-se caules com presença ou ausência de tecido medular, o que denominamos de caules cheios e ocos, respectivamente. 
Os caules, no início da idade de crescimento, para todas as variedades estudadas no presente trabalho, apresentaram-se com tecido medular. Após algum tempo, em maior ou menor número deles, o tecido medular começa a se contrair, dando origem a um vazio na sua região interior, que pode estender-se ou não, por todo o seu comprimento. Como todos os caules trabalhados tiveram, praticamente, a mesma idade de crescimento, é de supor que esta não seja a causa principal dessa ocorrência, podendo-se atribuí-la a fatores genéticos próprios de cada variedade.

Os caules das variedades de rami com maior quantidade de caules ocos são descorticados mais facilmente, além de permitir maior rendimento econômico de fibra. Além disso, as fibras são mais limpas, pois estão isentas dos restos do tecido medular que, em maior quantidade, acham-se aderidos às fibras obtidas dos caules cheios.

Por outro lado, a presença do tecido medular aumenta a resistência dos caules à ação dos ventos. As variedades de maior porcentagem de caules ocos são mais suscetíveis de terem os caules quebrados, acamados e emaranhados pelos ventos, o que dificulta a sua colheita, tanto manual como mecânica. Apesar de importante, esse problema pode ser contornado pelo ramicultor, que devz estabelecer seu ramizal em lugares naturalmente protegidos dos ventos fortes ou, então, defendê-lo por meio de fileiras de árvores, estrategicamente localizadas.

$\mathrm{Na}$ literatura especializada sobre rami, nada se encontrou referente aos assuntos acima mencionados. Sabe-se que os plantadores de rami, tendo um conhecimento prático sobre ele, dão preferência ao plantio de variedades que apresentam maior número de caules ocos, pois acham que os riscos do acamamento dos caules são compensados pela maior facilidade de trabalho da máquina descorticadora e conseqüente maior rendimento em fibras.

Visando determinar possíveis variações em características gerais dos caules de rami decorrentes da presença ou não do tecido medular, foi efetuado um levantamento comparativo em dez variedades da coleção do I.A.C. Os resultados são relatados no presente trabalho.

\section{MATERIAL E MÉTODOS}

Para o presente estudo, aproveitou-se o mesmo material utilizado para a elaboração de trabalhos anteriores $(\mathbf{1}, \mathbf{2})$. Utilizaram-se as variedades internacionais de rami (Boehmeria nivea Gaud.): saikeiseishin-shun, london, tatsutyama, izu-kairyo, chiyai, tsuannan n. 2 , toakan, kokuhi E 49-63 e tatsuka-kairyo, cuja forma de obtenção e origem estão mencionadas em trabalho anterior (2).

$\mathrm{Na}$ ocasião do corte dos pedaços dos caules de cada variedade, estes foram separados em dois tipos: caule cheio, com a presença do tecido medular em toda a sua região interior; caule oco, com o tecido medular contraído, dando origem a um vazio na sua região interior.

O método utilizado na obtenção das amostras dos caules e de fibras foi o mesmo relatado pelo autor em outros trabalhos $(\mathbf{1}, \mathbf{2})$. 
Foram calculados os intervalos de confiança para as médias das diferentes características de cada variedade, pelo teste de "student" ao nível de significância de $5 \%$.

\section{RESULTADOS E DISCUSSÃO}

No quadro 1, estão indicadas as porcentagens obtidas do número de caules cheios para as dez variedades de rami estudadas, lembrando que foram trabalhados, para cada variedade, sessenta caules, correspondendo a três caules, escolhidos ao acaso, de vinte plantas.

Os números mostram que enquanto algumas variedades apresentaram porcentagens elevadas de caules cheios (izu-kairyo e tsuannan n. ${ }^{\circ} 2$, com 76,6 e $85,0 \%$ respectivamente), outras apresentaram porcentagens elevadas de caules ocos (saikeiseishin-shun, chiayi e E 49-63, com 70,0,
78,3 e $86,7 \%$ respectivamente). As variedades london, tatsutyama e toakan, tiveram, praticamente, $50 \%$ de caules cheios e ocos.

Nos quadros 2 e 3 , acham-se as médias e os intervalos de confiança das características estudadas nos caules cheios e ocos das dez variedades de rami em estudo.

Para as características de peso, os coeficientes de variação foram de 0,0 a $59,6 \%$, o que indica que as variedades podem responder favoravelmente a uma seleção quanto a essas características, sendo que a tsuannan n. 2 foi a que apresentou coeficientes de variação mais altos.

Com relação às características de teor, comprimento e fineza de fibra, diâmetro e comprimento dos caules, os coeficientes de variação foram baixos $(6,9$ a $15,6 \% ; 4,3$ a $9,6 \% ; 9,9$ a $22,4 \% ; 7,3$ a $18,0 \%$, ะ 7,4 a $23,1 \%$, respectivamente), indicando que, pro-

QUADRO 1. - Porcentragem de caules cheios em dez variedades de rami

\begin{tabular}{|c|c|}
\hline & $\%$ \\
\hline Saikeiseishin-shun & 30,0 \\
\hline London $\ldots . .$. & 48,3 \\
\hline Tatsutyama & 53,3 \\
\hline Izu-kairyo $\ldots \ldots \ldots \ldots \ldots \ldots \ldots \ldots \ldots \ldots \ldots \ldots \ldots \ldots \ldots \ldots \ldots \ldots \ldots \ldots \ldots \ldots \ldots$ & 76,6 \\
\hline Chiayi $\ldots \ldots \ldots \ldots \ldots \ldots$ & 217 \\
\hline Tsuannan $n .^{\circ} 2$ & 85,0 \\
\hline 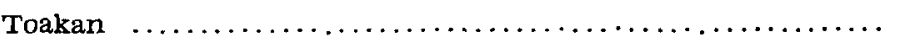 & 51,7 \\
\hline$\ldots \ldots \ldots \ldots \ldots \ldots \ldots$ & 63,3 \\
\hline E $49-63 \ldots \ldots \ldots$ & 13,3 \\
\hline Tatsuka-kairyo & 61,7 \\
\hline
\end{tabular}




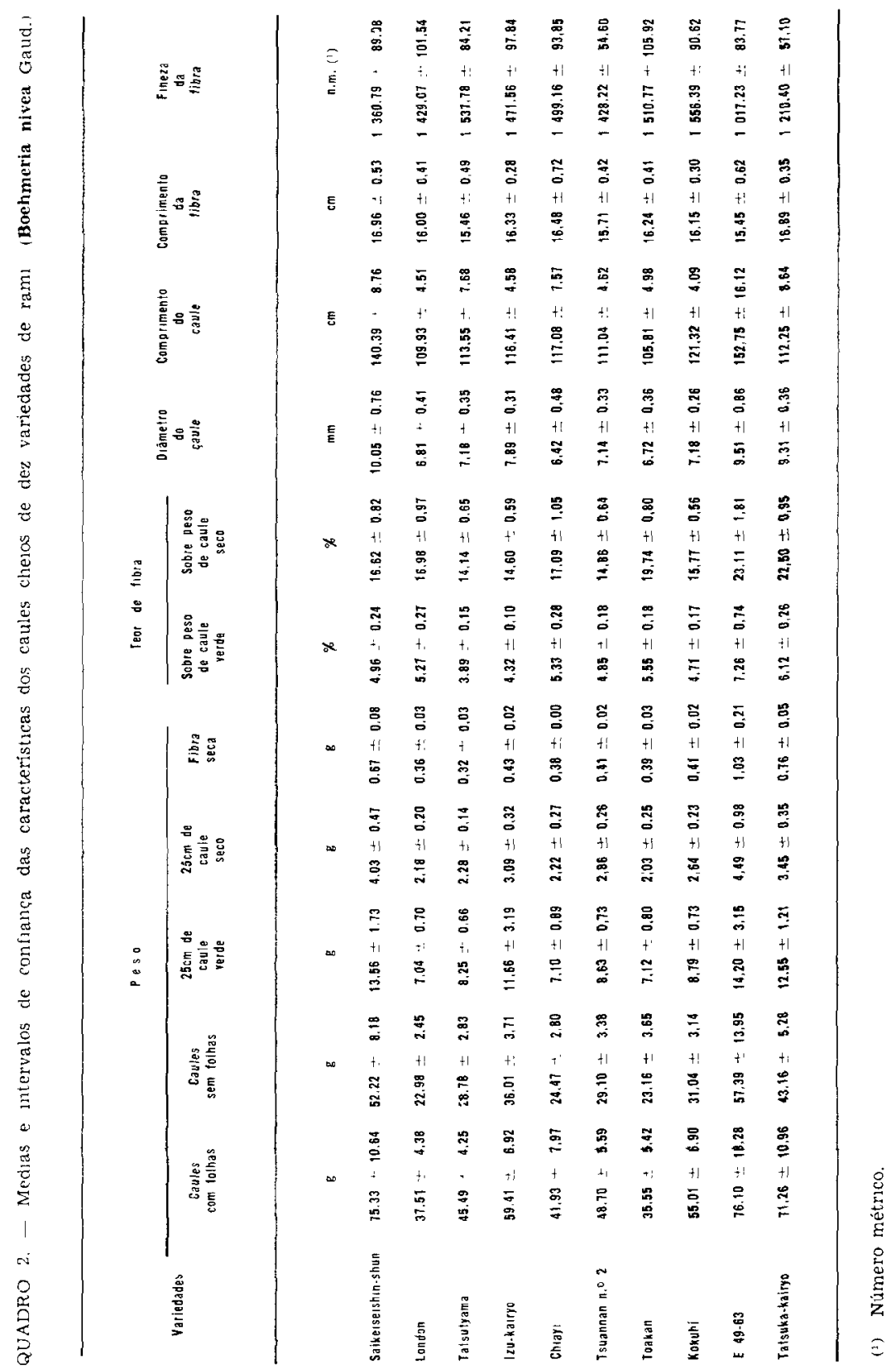




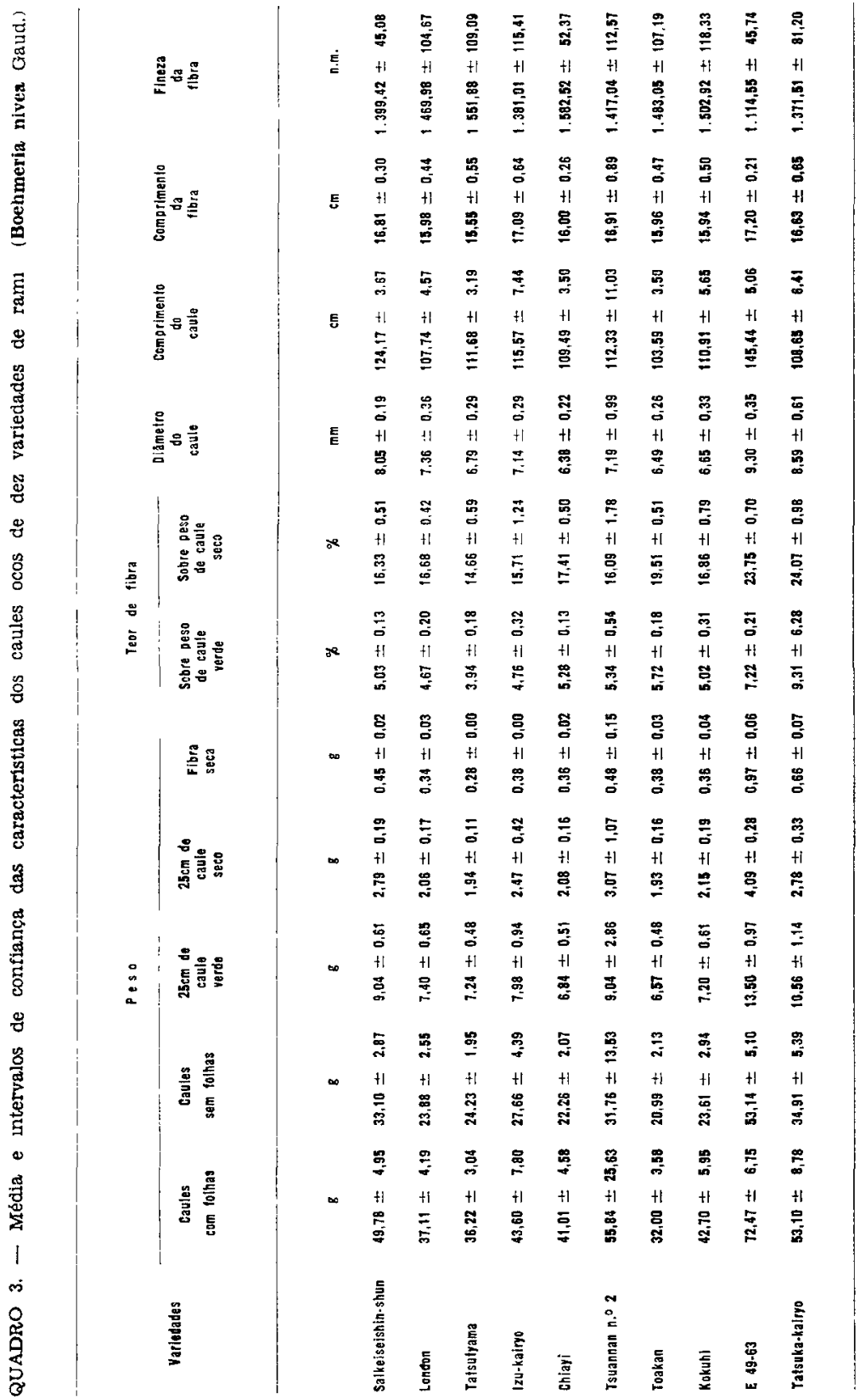


vavelmente, as variedades já tenham sido submetidas a seleções anteriores, sendo esperadas dificuldades em trabalhos de seleção que visem ao melhoramento dessas características, principalmente da segunda mencionada.

\subsection{VARLAÇ̃O DAS}

CARACTERISTICAS DENTRO DE

VARIEDADES, DETERMTNADAS

PARA CAULES CHEIOS E OCOS

A variedade saikeiseishin-shun apresentou médias superiores para os caules cheios em relação aos ocos para as características de peso de caules com folhas e sem folhas, peso de $25 \mathrm{~cm}$ de caule verde e seco, peso de fibra seca, diâmetro e comprimento de caule $(75,33$ e $49,78 \mathrm{~g} ; 52,22$ e $33,10 \mathrm{~g} ; 13,56$ e $9,04 \mathrm{~g} ; 4,03$ e $2,79 \mathrm{~g}$; 0,67 e $0,45 \mathrm{~g} ; 10,05$ e $8,05 \mathrm{~mm}$ e 140,39 e $124,17 \mathrm{~cm}$ respectivamente). Para as características de porcentagens, comprimento e fineza de fibra, as médias estiveram dentro dos intervalos de confiança $(4,96$ e $5,03 \%$; 16,62 e $16,33 \% ; 16,96$ e $16,81 \mathrm{~cm}$; $1.360,79$ e $1.399,42$ n.m., respectivamente).

A variedade london apresentou médias estatisticamente diferentes somente com relação à porcentagem de fibra sobre peso de caule verde em que os caules cheios foram superiores (5,27 e 4,67\% respectivamente). Para as características restantes, não houve diferenças entre as médias de caule cheio e oco.

Quanto à variedade tatsutyama, as médias encontradas para os caules cheios com relação às características de peso de caules com folhas, peso de $25 \mathrm{~cm}$ de caule verde e peso de fibras secas, foram superiores às encontradas para os caules ocos $(45,49$ e $36,22 \mathrm{~g} ; 8,25$ e $7,24 \mathrm{~g} ; 0,32$ e $0,28 \mathrm{~g}$ respectivamente). Nas características restantes, as médias estiveram dentro dos intervalos de confiança.

Para a variedade izu-kairyo, as características de peso de caules com folhas e sem folhas, peso de fibras secas e diâmetro dos caules apresentaram médias superiores para os caules cheios com relação aos ocos $(59,41$ e $43,60 \mathrm{~g} ; 36,01$ e $27,66 \mathrm{~g} ; 0,43$ e $0,38 \mathrm{~g} ; 7,89$ e $7,14 \mathrm{~mm}$ respectivamente). Nas outras características, as médias não diferiram.

As variedades chiayi, tsuannan n. ${ }^{\circ} 2$, toakan e E 49-63 não apresentaram diferenças para as médias das características estudadas com respeito aos caules cheios e ocos.

As médias encontradas para os caules cheios da variedade kokuhi, com relação às características de peso de caules sem folhas, peso de $25 \mathrm{~cm}$ de caules verdes e secos e comprimento do caule foram superiores às das encontradas para us caules ocos $(31,04 \mathrm{e}$ $23,61 \mathrm{~g} ; 8,79$ e $7,20 \mathrm{~g} ; 2,64$ e 2,15g; 121,32 e $110,91 \mathrm{~cm}$ respectivamente). Nas características restantes, as médias estiveram dentro dos intervalos de confiança, para os dois tipos de caules considerados.

Com relação à variedade tatsuka-kairyo, as médias encontradas para as características dos caules cheios e ocos não diferiram estatisticamente, com exceção da fineza, em que a média dos caules ocos foi superior à dos caules cheios $(1.371,51$ e $1.210,40$ n.m., respectivamente).

\subsection{COMPARAÇÃO ENTRE VARIEDADES PARA CAULES CHEIOS}

Comparando-se duas a duas, estatisticamente, as médias das caracte- 
rísticas encontradas para caules cheios das variedades estudadas (quadro 2), verifica-se que, para peso de caules com folhas e sem folhas, a variedade E 49-63 apresentou as maiores médias $(76,10$ e $57,39 \mathrm{~g}$, respectivamente), que foram superiores às de todas as variedades restantes, com exceção das variedades saikeiseishin-shun e tatsuka-kairyo, cujas médias não diferiram $(75,33$ e $52,22 \mathrm{~g}$ para a primeira e 71,26 e 43,16g para a segunda respectivamente).

Quanto ao peso de $25 \mathrm{~cm}$ de caule verde, a variedade $E$ 49-63 teve uma média $(14,20 \mathrm{~g})$ superior a todas as variedades restantes, mas não diferiu das encontradas para as variedades saikeiseishin-shun, tatsuka-kairyo e izu-kairyo $(13,56 ; 12,55$ e $11,66 \mathrm{~g}$, respectivamente).

Com respeito ao peso de $25 \mathrm{~cm}$ de caule seco, a E 49-63 teve a maior média $(4,49 \mathrm{~g})$, que foi superior a todas as outras variedades, sem diferir da saikeiseishin-sun $(4,03 \mathrm{~g})$ e tatsuka-kairyo $(3,45 \mathrm{~g})$.

Considerando o peso de fibra seca, a variedade $E$ 49-63, com uma média de $1,03 \mathrm{~g}$, foi superior a todas as outras variedades estudadas.

Quanto à porcentagem de fibra sobre peso de caule verde, a E 49-63, com uma média de $7,26 \mathrm{~g}$, foi superior a todas as outras variedades.

Com relação à porcentagem de fibra sobre peso de caule seco, as variedades E 49-63 e tatsuka-kairyo tiveram médias de 23,11 e $22,50 \mathrm{~g}$ respectivamente, dentro dos mesmos intervalos de confiança, sendo superiores às de todas as outras variedades consideradas.
As porcentagens de fibra, tanto sobre peso de caule verde como seco, têm sido utilizadas, pela maior parte dos melhoristas de rami, para avaliar a riqueza de fibra da planta. Examinando-se as médias obtidas para peso de fibra seca, que realmente representa a riqueza de fibra, e as obtidas pelas porcentagens de fibra, verifica-se que há certa correspondência de valores, o que indica que esses últimos métodos, apesar de apresentarem algumas discrepâncias para algumas variedades, podem ser utilizados, devido às facilidades de execução, em uma fase preliminar de seleção do rami. A seleção final deve sempre utilizar o método da quantidade real de fibra nos caules, que, apesar do exigir maior e mais cuidadoso trabalho, oferece valores mais precisos.

Para a característica de diâmetro do caule, as médias das variedades E 49-63, tatsuka-kairyo e saikeiseishin-shun, não diferiram entre si $(9,51 ; 9,31$ e $10,05 \mathrm{~mm}$ respectivamente), mas foram superiores a todas as outras variedades consideradas. $\mathrm{Ob}$ servando-se as médias de diâmetro $e$ as de fineza de fibra, nota-se que as variedades de caules mais grossos foram as que apresentaram as fibras mais grossas, concordando com o que foi obtido por CIARAMELLO; MEDINA \& SALGADO (3), ao concluir que os caules de menor diâmetro apresentam fibras mais finas. Nota-se, também, que as variedades de caules mais grossos foram as que produziram as maiores quantidades de fibras.

Quanto ao comprimento do caule, as variedades E 49-63 e saikeiseishin-shun, com médias de 152,75 e $140,39 \mathrm{~cm}$ respectivamente, não diferiram entre si, mas foram superiores 
a todas as outras variedades estudadas.

Com respeito ao comprimento de fibra, a saikeiseishin-shun apresentou a maior média $(16,96 \mathrm{~cm})$, que foi superior às médias da london, tatsutyama, tsuannan n. ${ }^{\circ} 2(16,00 ; 15,46 \mathrm{e}$ $15,71 \mathrm{~cm}$ respectivamente), não diferindo das médias das variedades restantes.

Com relação à fineza de fibra, a variedade de fibra mais grossa foi a E 49-63, com um índice de fineza de $1.017,23$ n.m., inferior ao de todas as outras variedades em estudo. As variedades kokuhi, tatsutyama e toakan apresentaram as fibras mais finas, com índices de fineza de 1556,39; $1.537,78$ e $1.510,77$ n.m. respectivamente.

3.3. COMPARAC̄ÃO ENTRE VARIEDADES PARA CAULES OCOS

Comparando-se duas a duas, estatisticamente, as médias encontradas para as características dos caules ocos das variedades em estudo (quadro 3), verifica-se que, para peso de caules com folhas e peso de caules sem folhas, a variedade E 49-63, com médias de 72,47 e $53,14 \mathrm{~g}$, respectivamente, foi superior a todas as outras. Entre estas, as de maiores médias foram a saikeiseishin-shun, tsuannan n. ${ }^{\circ} 2$ e tatsuka-kairyo, com 49,78 e $33,10 \mathrm{~g} ; 55,84$ e $31,76 \mathrm{~g}$ e 53,10 e $34,91 \mathrm{~g}$, respectivamente, para peso de caules com folhas e sem folhas.

Considerando os pesos de $25 \mathrm{~cm}$ de caule verde e seco, a E 49-63, com médias de 13,50 e $4,09 \mathrm{~g}$, respectivamente, foi superior a todas as outras, salientando-se entre estas, a tatsuka-kairyo, tsuannan n. ${ }^{\circ} 2$ e saikeiseishin-shun com médias de 10,56 e
2,$78 ; 9,04$ e 3,07 e 9,04 e $2,79 \mathrm{~g}$, respectivamente, para peso de caule verde e seco.

Com relação ao peso de fibra seca, a E 49-63, com uma média de $0,97 \mathrm{~g}$, foi superior a todas as outras. As variedades tatsuka-kairyo, tsuannan $n .^{\circ} 2$ e saikeiseishin-shun foram, a seguir, as que apresentaram as maiores médias $(0,66 ; 0,48$ e $0,45 \mathrm{~g}$, respectivamente) de fibras secas.

Quanto ao teor de fibra sobre peso de caule verde e peso de caule seco, as variedades tatsuka-kairyo e E 49-63 com médias dentro do mesmo intervalo de confiança 9,31 e $7,22 \%$ para o primeiro e 24,07 e $23,75 \%$ para o segundo, respectivamente, foram superiores a todas as outras.

Com respeito ao diâmetro do caule, as variedades $\mathrm{E}$ 49-63, com média $9,30 \mathrm{~mm}$, e tatsuka-kairyo, com $8,59 \mathrm{~mm}$, foram superiores a todas as outras.

Com relação ao comprimento do caule, a $E$ 49-63, com uma média de $145,44 \mathrm{~cm}$, foi superior a todas as outras, vindo a seguir a saikeiseishin-shun, izu-kairyo e tsuannan n. ${ }^{\circ} 2$, com médias de 124,$17 ; 115,57$ e $112,33 \mathrm{~cm}$ respectivamente.

Para comprimento de fibra, as variedades que tiveram as fibras mais longas foram E 49-63, izu-kairyo, tsuannan n. 2 a saikeiseishin-shun, com médias de 17,20,17,09, 16,91 e 16,81 respectivamente, situadas no mesmo intervalo de confiança.

A E 49-63 apresentou a fineza de fibra com uma média de 1.114,55 n.m., inferior ao índice de todas as outras variedades em estudo, sendo, portanto, a de fibras mais grossas, pois, quanto menor o índice de fineza, 
mais grossa é a fibra. As variedades chiayi, tatsutyama e kokuhi apresentaram as fibras mais finas, com indices de fineza de $1.582,52 ; 1.551,88 \mathrm{e}$ $1.502,92$ n.m. respectivamente.

Verifica-se, pelo presente estudo, que as variedades $E$ 49-63, tatsuka-kairyo e saikeiseishin-shun, tanto para caules cheios como para ocos apresentaram os melhores valores para as características estudadas, com exceção da fineza de fibra, em que os valores foram os mais baixos. Em um projeto de melhoramento do rami, deve-se lançar mão dessas variedades de acordo com a finalidade de utilização da fibra. Para a confecção de aniagens, cortinas, cordas, barbantes e tapetes onde são utilizadas fibras mais grossas, as variedades mencionadas poderão entrar no trabalho do melhorista. Mas, para a confecção de fios mais finos destinados a compor diversos tecidos mais nobres como roupa de vestuário, de cama e de mesa, deve o melhorista utilizar-se, em seu trabalho de melhoramento, de variedades de fibra mais finas como a chiayi, tatsutyama, toakan e kokuhi.

\section{CONCLUSÕES}

a) As variedades apresentaram uma maior ou menor porcentagem de caules cheios ou ocos. b) Algumas variedades revelaram superioridade dos caules cheios em relação aos ocos quanto às características de peso, porcentagem de fibra sobre peso de caule verde, diâmetro e comprimento de caule.

c) Para todas as variedades estudadas, não houve diferenças entre caules cheios e ocos quanto às características de porcentagem de fibra sobre peso de caule seco e comprimento de fibra.

d) Com respeito à fineza de fibra, apenas uma variedade (tatsuka-kairyo) apresentou superioridade dos caules ocos sobre os cheios.

e) Quando se consideraram separadamente os caules cheios e ocos, para cada variedade, comparadas duas a duas, tiveram comportamento diferente com relação às características estudadas.

f) As variedades saikeiseishin-shun, E 49-63 e tatsuka-kairvo, cujos caules cheios e ocos apresentaram os maiores valores para as características estudadas, com exceção de fineza de fibra, que teve os valores mais baixos, devem ser consideradas em futuros trabalhos de melhoramento do rami.

g) O estudo dos resultados revela que é possível efetuar um trabalho de melhoramento visando aperfeiçoar determinadas características do rami.

\section{CHARACTERISTICS VARIATION OF RAMI IN FUNCTION OF THE STEAM PITH NATURE}

\section{SUMMARY}

A study concerning some characters of ten ramie varieties (Boehmeria nivea Gaud.) and its relationship with the presence or absense of stalk pith is reported. The following international varieties were considered: saikeiseishin-shun, london, tatsutyama, izu-kairyo, chiayi, tsuannan n. ${ }^{\circ}$, toakan, koxuhi, E 49-63 and tatsuka-kairyo. 
The characters studied were: weight-green stalk, dry stalk, dry fibers, fiber content in green and dry stalk, diameter and length of stalk and fineness and length of fibers.

In some varieties, the stalks with pith showed superior performance with relation to characters of weight, fiber content in green stalk, diameter and length of stalk.

For all varieties, no differences were observed for the characters of fiber content in dry stalk and length of fibers in respect to the presence or absence of stalk pith.

For the character fineness of fiber, only one variety (tatsuka-kairyo) presented superior performance for the stalks with absence of pith.

When the varieties were confronted two and two, such for the presence or absence of stalk pith, they showed different behaviour in relation to the characters studied.

The stalks with presence or absence of pith of varieties Saikeiseishin-shun, E 49-63 and Tatsuka-kairyo, presented higher values for the characters tested, with except for fineness of fibers whose values were the lowest and could be considered in the future breeding program.

The results obtained show that it is possible to make a selection work with the objective to obtain certain characteristics of rami.

\section{LITERATURA CITADA}

1. CASTRO, G. A. P. Correlaçōes entre caracteristicas agrobotânicas e tecnológicas de dez variedades de rami. Bragantia, Campinas, 37:LXXXIX-XCIV, 1978. (Nota 16)

2. Fineza e comprimento de fibra de dez variedades de rami. Bragantia, Campinas, 35:155-162, 1976.

3. Ciaramello, D.; MEDiNA, J. C. \& SALGADO, A. L. B. Comprimento e diâmetro dos caules de rami e as caracteristicas da fibra. Bragantia, Campinas, 22:73-80, 1963. 